\title{
Evaluation of cell proliferation and apoptosis in Helicobacter pylori gastritis using an image analysis processor
}

\author{
Yasuo Ohrura ${ }^{1}$, Tadashi Furihata ${ }^{1,2}$, Hitoshi Kawamata ${ }^{1}$, Masafumi Tabuchi ${ }^{3}$, Keilchi Kubota ${ }^{2}$, \\ Akira Terano ${ }^{4}$, Taro SAKai ${ }^{1,4}$, and TAKahiro Fujimori ${ }^{1}$ \\ ${ }^{1}$ Department of Surgical and Molecular Pathology, Dokkyo University School of Medicine, 880 Kitakobayashi, Mibu, Shimotsuga, Tochigi \\ 321-0293, Japan \\ ${ }^{2}$ Department of Gastroenterological Surgery, Dokkyo University School of Medicine, Tochigi, Japan \\ ${ }^{3}$ Nakameguro Digestive Disease Clinic, Tokyo, Japan \\ ${ }^{4}$ Department of Gastroenterology, Dokkyo University School of Medicine, Tochigi, Japan
}

\begin{abstract}
Background. Infection of the gastric mucosa by Helicobacter pylori is primarily responsible for gastritis, gastric ulcer, adenocarcinoma, and lymphoproliferative disorders. $H$. pylori appears to accelerate apoptosis and the proliferation of the gastric epithelium directly or indirectly. To precisely assess the proliferative and apoptotic profile of $\mathrm{H}$. pylori-infected gastric mucosa, a quantitative imaging system is now required. Methods. Fifty-two patients with $\boldsymbol{H}$. pylori gastritis were the subjects of the study. Biopsy materials were taken from at least two sites (usually three to five sites) including the antrum and corpus. The grade of gastritis was evaluated by the Updated Sydney System. The proliferative and apoptotic profile was examined by Ki-67 immunohistochemistry and by a terminal deoxynucleotidyl transferase (TdT)-mediated dUTP nick end-labeling method. In addition, Ki-67-positive cells were quantitated by an image processor for analytical pathology (IPAP) system.

Results. $\boldsymbol{H}$. pylori density and polymorphonuclear neutrophil activity were significantly decreased after $H$. pylori eradication $(P<0.0001)$. Chronic inflammation $(P<0.0001)$ and lymphoid follicle numbers $(P<0.0005)$ were also significantly decreased after the eradication. Glandular atrophy and intestinal metaplasia were slightly decreased after eradication, but the decrease did not reach the significant level. The Ki-67 labeling index was significantly decreased after the eradication $(P<0.0001)$. The apoptosis index was also decreased after the eradication, but this decrease did not reach the significant level $(P=0.06)$.

Conclusion. Our data suggest that the activation of proliferative cells and induction of apoptosis in the gastric mucosa is a response to $\boldsymbol{H}$. pylori-induced mucosal damage. Moreover, IPAP may be a useful technology for evaluating the results of immunohistochemistry, and it could provide quantitative and reliable data for studying $H$. pylori gastritis.
\end{abstract}

Offprint requests to: T. Fujimori

The first two authors contributed equally to this study.

Received: July 22, 2002 / Accepted: October 10, 2002
Key words Helicobacter pylori gastritis · Apoptosis · Ki-67 · Cell proliferation - Image analysis processor

\section{Introduction}

Helicobacter pylori infection of the gastric mucosa is primarily responsible for gastritis [1], and has been reported to be implicated in various gastrointestinal diseases, such as gastric ulcer, adenocarcinoma and lymphoproliferative disorders (mucosa-associated lymphoid tissue [MALT]oma) [1-6]. H. pylori-infected gastric mucosa shows infiltration of polymorphonuclear leukocytes, lymphocytes, monocytes, and plasma cells in the lamina propria, and intraepithelial neutrophil infiltration [7]. Severe intraepithelial neutrophil infiltration is a characteristic feature of $H$. pylori gastritis, and is correlated with the mucosal damage that is due to the effects of various cytokines, free radicals, and monochloramine [8]. These changes would accelerate apoptosis and proliferation in the foveolar neck epithelium.

$\mathrm{Ki}-67$ is a nuclear antigen that is expressed in all phases of the cell cycle except for G0 phase, and it has been used as a marker for proliferating cells [9]. A monoclonal antibody against Ki-67 (MIB-1) is widely used for immunohistochemistry to measure the proliferative ratio in normal tissue, inflammatory tissue, and tumor tissue [9]. However, in most of the previous experiments, Ki-67-positive cells were counted by pathologists by microscopy [10]. Now, a quantitative imaging system is required for counting Ki-67-positive cells.

In this study, we examined the proliferative and apoptotic profile of $H$. pylori-infected gastric mucosa before and after $H$. pylori eradication, by using $\mathrm{Ki}-67$ immunohistochemistry and a terminal deoxynucleotidyl transferase (TdT)-mediated dUTP nick endlabeling 
(TUNEL) method. We utilized an image processor for analytical pathology (IPAP) [11] to quantitate the Ki67-positive cells.

\section{Patients, materials, and methods}

\section{Patients}

Gastric mucosa was obtained from 52 Japanese patients with $H$. pylori gastritis (39 men and 13 women; mean age, 58.7 years; range, 43 to 83 years) during the period from January to August, 1997. Biopsy materials were taken from at least two sites (usually three to five sites according to the Updated Sydney System), including the antrum and corpus. All of the patients were tested by the rapid urease test (CLO test) for $H$. pylori infection. The presence of $H$. pylori in the patients was also confirmed by histological examination. After H. pylori eradication was carried out with clarithromycin and metronidazole, the patients were examined again for $H$. pylori infection by the CLO test and by histological examination. Then only the patients who were negative for $H$. pylori infection over a 3-month period were the subjects of the study.

\section{Tissue preparation}

Biopsy specimens were fixed in $10 \%$ formalin and embedded in paraffin. Two-micrometer-thick sections were stained with hematoxylin-eosin or Giemsa for histological examinations. Four-micrometer-thick sections were used for immunohistochemistry and the TUNEL method.

\section{Assessment of gastritis}

The specimens were examined independently by two experienced pathologists (Y.O., T.F.). The histological grading of gastritis before and after eradication was evaluated according to the updated Sydney System [12]. A four-point scale $(0$, normal; 1 , mild; 2 , moderate; and 3 , marked) was used for evaluating graded variables, such as $H$. pylori density, polymorphonuclear neutrophil activity, chronic inflammation, glandular atrophy, and intestinal metaplasia, and a nongraded variable, lymphoid follicle. We took biopsy materials from two to five sites in the stomach, and evaluated the score from the specimen showing the highest histological grading. After taking the grading scores for all variables in the entire area of the selected specimen, the scores were averaged for each variable.

\section{TUNEL method}

For the detection of apoptotic cells, the TUNEL method (Apoptosis in situ Detection Kit; Wako, Osaka, Japan) was used [13]. After routine deparaffinization, the section was incubated with proteinase for $5 \mathrm{~min}$ at $37^{\circ} \mathrm{C}$, and treated with biotin-labeled dUTP and $\mathrm{TdT}$ for $10 \mathrm{~min}$ at $37^{\circ} \mathrm{C}$. The section was washed three

Table 2. Ki-67 labeling index and apoptotic index values before and after $H$. pylori eradication

\begin{tabular}{lcc}
\hline & $\begin{array}{c}\text { Before } \\
\text { eradication }(\%)\end{array}$ & $\begin{array}{c}\text { After } \\
\text { eradication }(\%)\end{array}$ \\
\hline Ki-67 labeling index & 11.78 & $0.98^{*}$ \\
Apoptotic index & 2.05 & $1.07 * *$ \\
* Ki-67 labeling index was significantly decreased by the eradication \\
$(P<0.0001)$
\end{tabular}

Table 1. Histological evaluation before and after eradication of Helicobacter pylori

\begin{tabular}{lcc}
\hline & \multicolumn{2}{c}{ Grade of gastritis $^{\mathrm{a}}$} \\
\cline { 2 - 3 } & Before eradication & After eradication \\
\hline Helicobacter pylori density & 1.57 & $0.21^{*}$ \\
Neutrophil activity & 1.82 & $0.50^{*}$ \\
Mononuclear cell activity & 1.78 & $1.12^{*}$ \\
Lymphoid follicle number & 0.90 & $0.35^{*}$ \\
Glandular atrophy & 0.44 & 0.24 \\
Intestinal metaplasia & 0.20 & 0.10 \\
\hline * $P<0.0001 ; * * P<0.0005$ & \\
Helicobacter pylori density and neutrophil activity were significantly decreased by $H$. pylori \\
eradication. Mononuclear cell activity and lymphoid follicle numbers were also decreased \\
significantly after eradication. Glandular atrophy and intestinal metaplasia were slightly \\
decreased after eradication, but the decrease did not reach the significant level \\
a According to the Updated Sydney System [12] \\
b Although the presence of lymphoid follicles was classified as other histological features (non \\
graded variables) in the Updated Sydney System, we counted the number of the lymphoid follicles \\
in the same area of the biopsy materials before and after eradication
\end{tabular}


times for $5 \mathrm{~min}$ with $10 \mathrm{mM}$ phosphate-buffered saline ( $\mathrm{pH}$ 7.4; PBS), and then endogenous peroxidase was quenched with $3 \% \mathrm{H}_{2} \mathrm{O}_{2}$ in PBS for $5 \mathrm{~min}$ at room temperature. The section was then washed three times in PBS and incubated with peroxidase-conjugated antibiotin antibody for $10 \mathrm{~min}$ at $37^{\circ} \mathrm{C}$. The section was developed with 3,3-diaminobenizidine (DAB), and counterstained with methyl green.

Ten glands were selected randomly from each specimen, and at least 500 epithelial cells were counted for evaluating the induction of apoptosis. The number of positive cells per 500 cells at $\times 100$ magnification was expressed as the apoptotic index.

\section{Ki-67 immunohistochemistry}

After deparaffinization, the section was placed in $100 \%$ methanol with $0.3 \%$ hydrogen peroxide for $20 \mathrm{~min}$ to block endogenous peroxidase activity. To retrieve the antigen, the section was treated with microwave radiation (MI-77; Azuyama, Tokyo, Japan) for $10 \mathrm{~min}$ $(500 \mathrm{~W})$ and then allowed to cool for approximately $20 \mathrm{~min}$ at room temperature. A mouse monoclonal antibody against Ki-67 (MIB-1; Immunotech, Marseille, France) [14] was used as a primary antibody (diluted 1:50). The section was washed with PBS, incubated with biotinylated anti-mouse immunoglobulin for $15 \mathrm{~min}$ at room temperature and subsequently incubated with peroxidase-conjugated streptavidin for $10 \mathrm{~min}$ at room temperature (LSAB Kit; Dako, Kyoto, Japan). The section was again washed with PBS, and the antigenantibody complexes were visualized by DAB. The section was counterstained with hematoxylin. As negative controls, PBS and normal mouse IgG were substituted for the primary antibodies.

\section{Image analysis processor examination}

Positive staining for Ki-67 was quantitated by an image analyzer (image processor for analytical pathology; IPAP; Sumika Technos, Osaka, Japan). The image was produced from a light microscope with a $\times 40$ objective lens by the IPAP color television camera. The Ki-67 labeling index was determined by calculating the ratio of the area of positive nuclei to the area of all nuclei in three randomly selected areas.

\section{Statistical analysis}

The $\chi^{2}$ and Fisher's exact tests were applied to evaluate the significance of differences. $P$ values of less than 0.05 were considered statistically significant.
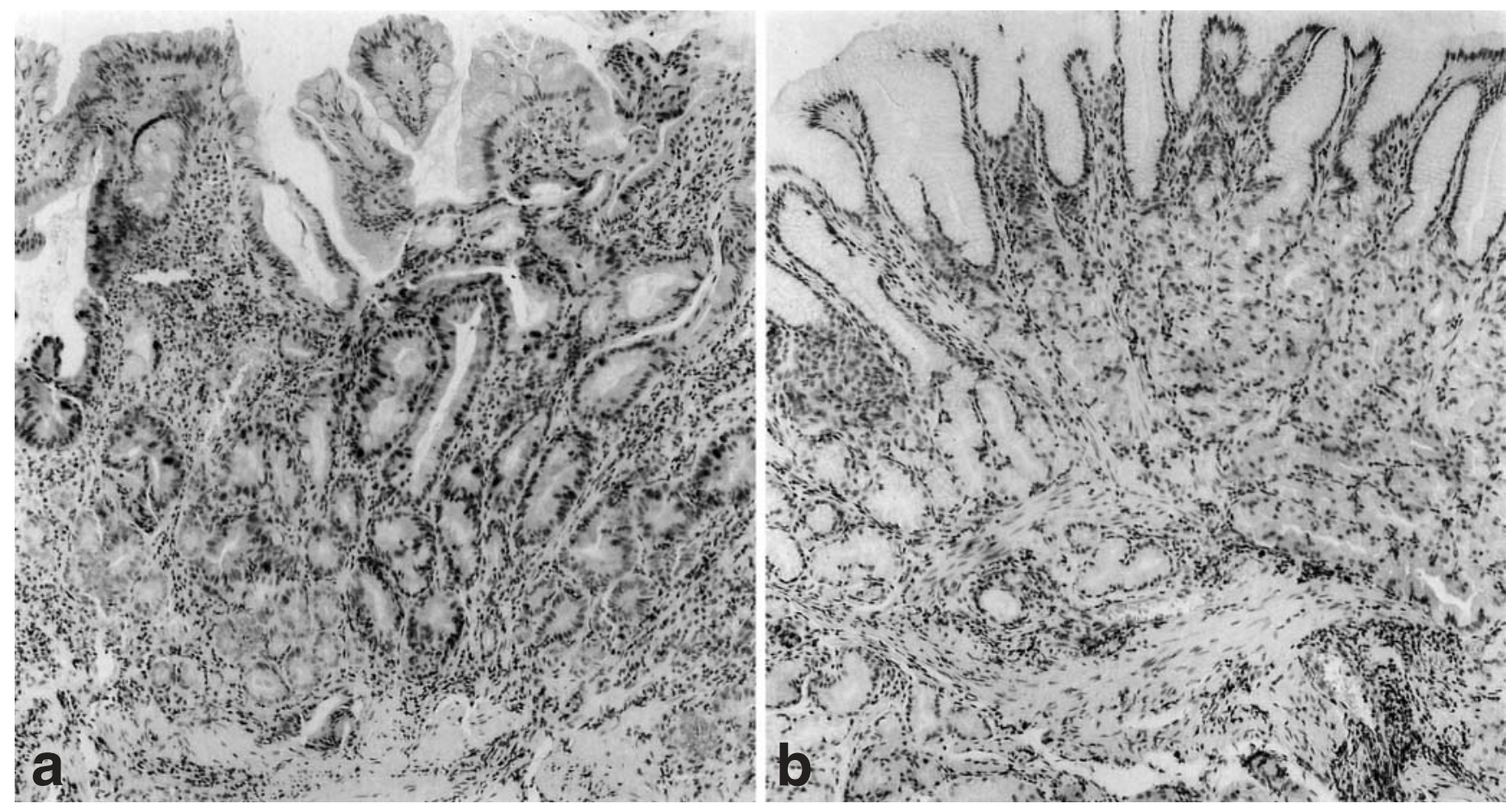

Fig. 1a,b. Ki-67 (MIB-1) immunohistochemistry. a Gastric mucosa showing severe active gastritis before Helicobacter pylori eradication. Ki-67 immunoreactivity is observed in the proliferative zone of the foveolar epithelium. b Ki-67-positive cells of the gastric mucosa after the eradication were decreased compared with those in the gastric mucosa before eradication. $\mathbf{a} ; \mathbf{b} \times 33$ 


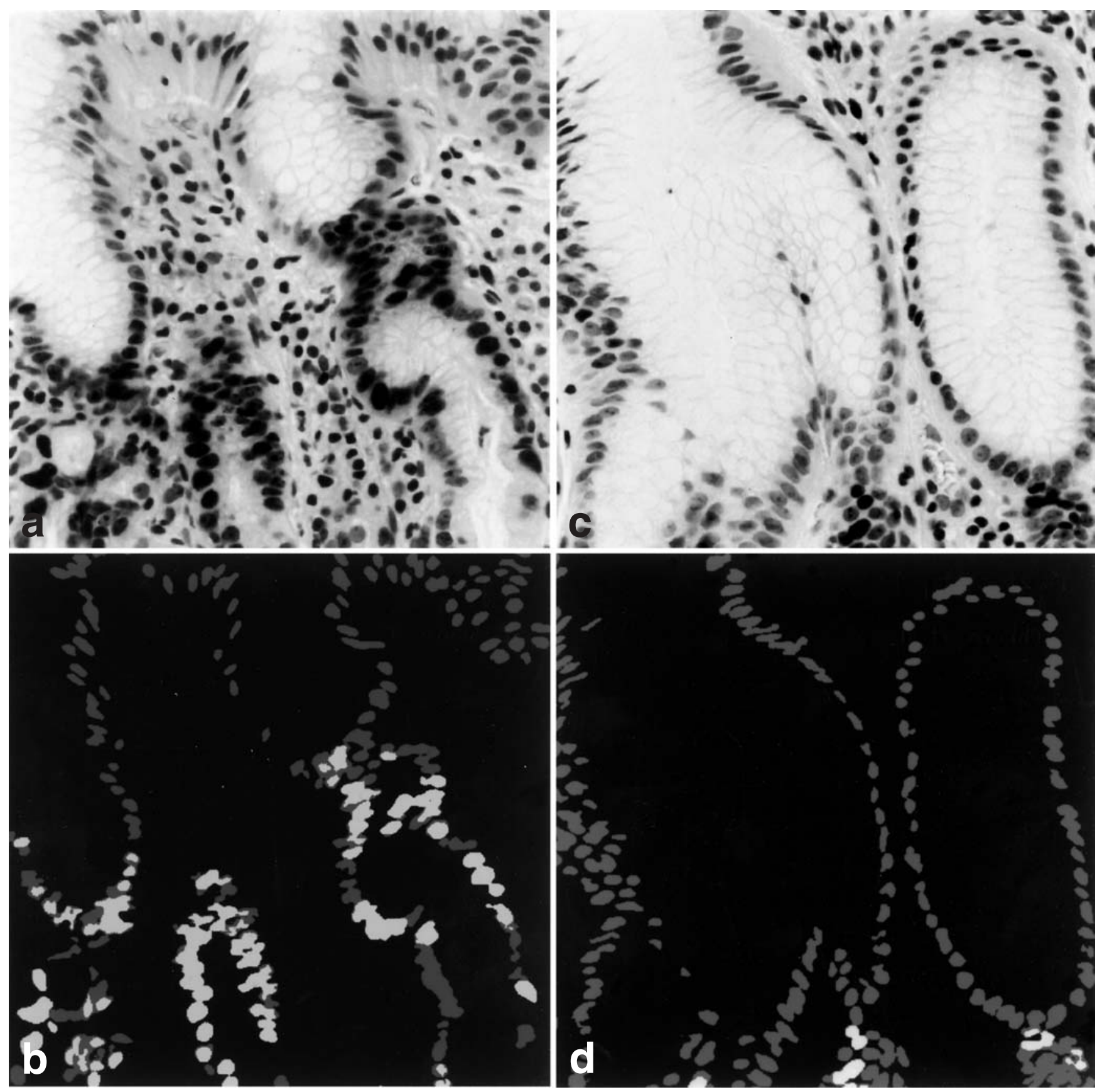

Fig. 2. a Gastric mucosa before H. pylori eradication, showing widely distributed Ki-67-positive cells. b Corresponding image analysis measuring the percentage of the area of positive-stained Ki-67 nuclei (white area)/total area of nuclei (white area and gray area). c Gastric mucosa after the eradication, showing scanty Ki-67-positive cells. d The area of positive-stained cells (white area) was markedly decreased compared with the area in the gastric mucosa before the eradication. $\mathbf{a}$ and $\mathbf{c} \times 40$

\section{Results}

\section{Classification and grading of gastritis}

The grades of gastritis before and after H. pylori eradication, based on the Updated Sydney System, are summarized in Table 1. H. pylori density was decreased from a grade of 1.57 to 0.21 , polymorphonuclear neutrophil activity from 1.82 to 0.50 , chronic inflammation (mononuclear cell activity) from 1.78 to 1.12 , glandular atrophy from 0.44 to 0.24 , intestinal metaplasia from 0.20 to 0.10 , and lymphoid follicle number from 0.90 to 0.35 after the eradication. H. pylori density and polymorphonuclear neutrophil activity were significantly 

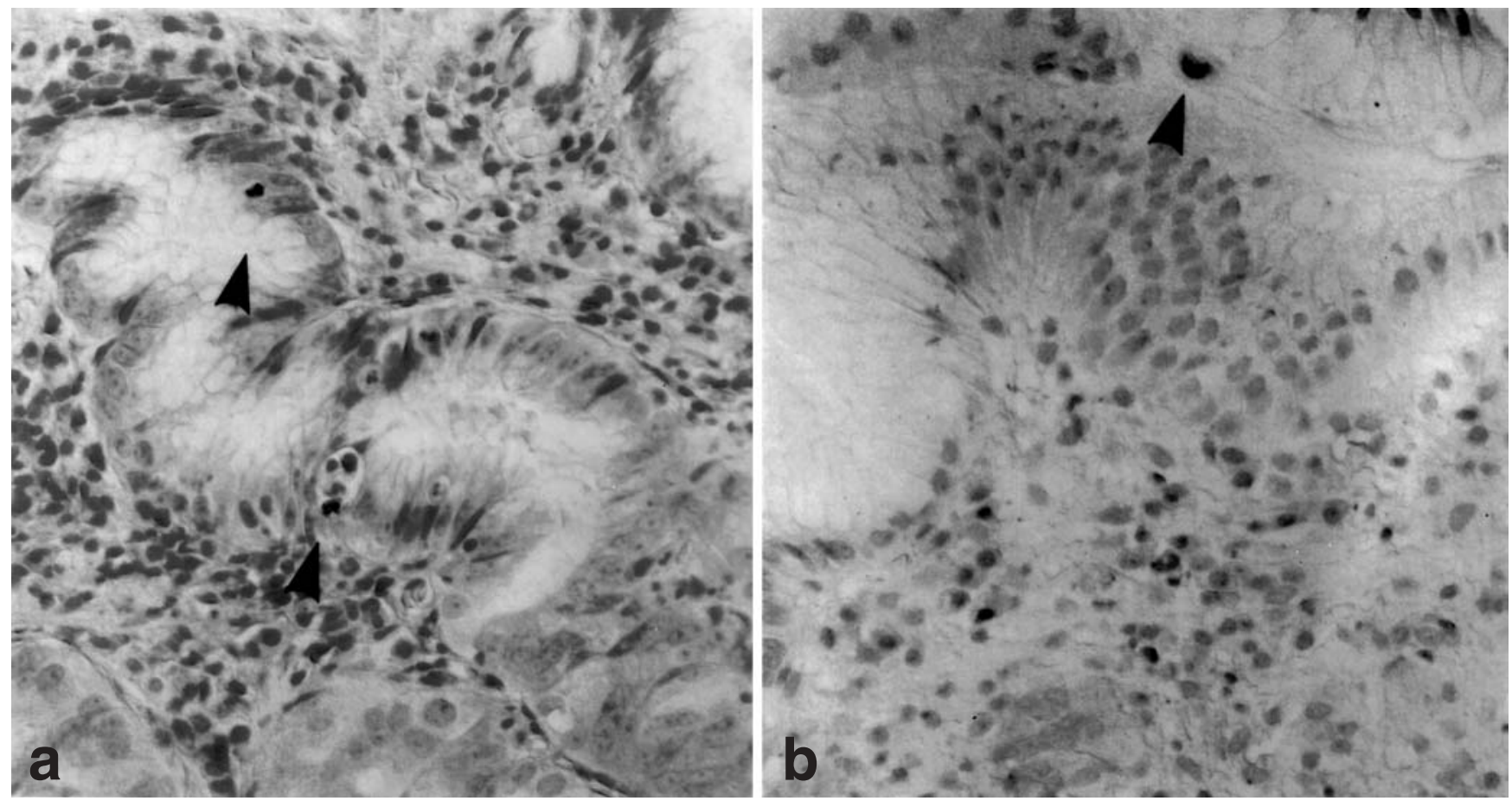

Fig. 3. a Terminal deoxynucleotidyl transferase-mediated dUTP nick end labeling (TUNEL)-positive cells (arrowheads) observed in the neck of the foveolar gland, equivalent to the proliferative zone (before H. pylori eradication). b Apoptotic cells (arrowhead) are observed to be normally distributed on the top of the superficial epithelium after the eradication. $\mathbf{a}$ and $\mathbf{b} \times 100$

decreased after eradication $(P<0.0001)$. Chronic inflammation $(P<0.0001)$ and lymphoid follicle number $(P<0.0005)$ were also significantly decreased after eradication. Glandular atrophy and intestinal metaplasia decreased slightly after eradication, but the decrease did not reach the significant level.

\section{Ki-67 immunostaining}

Ki-67-positive cells were localized in the proliferative zone in the gastric epithelium, and were diffusely distributed in the specimens with severe inflammation (Fig. 1a). However, Ki-67 was weakly positive in the glandular neck cells after $H$. pylori eradication (Fig. 1b). The Ki-67 labeling index, which was determined by IPAP, was significantly decreased after the eradication (Fig. 2a, b, c, d). The average value of the Ki-67 labeling index before eradication was $11.78 \%$, whereas the labeling index after eradication was $0.98 \%(P<0.0001$; Table 2). Thus, the IPAP data provided a clear image for evaluating the proliferative profile of $H$. pylori gastritis.

\section{Apoptosis}

The number of TUNEL-positive cells increased proportionally with the severity of the inflammatory grade, and
TUNEL-positive cells were observed even in the proliferative zone before $H$. pylori eradication (Fig. 3a). However, after the eradication, TUNEL-positive cells were present only on the superficial part of the epithelium, which is a typical pattern of normal mucosa (Fig. $3 b)$. The mean apoptotic index was decreased after the eradication, from $2.05 \%$ to $1.07 \%$, although the difference did not reach the significant level $(P=0.06$; Table 2).

\section{Discussion}

In this study, we showed a significant reduction of neutrophils and mononuclear cells in the gastric mucosa after $H$. pylori eradication in patients with $H$. pylori gastritis, and the reductions were associated with decreases in the Ki-67 labeling and apoptotic indices. Neutrophil activity is well known to be linked to the mucosal damage in $H$. pylori gastritis. Thus, the activation of proliferative cells and the induction of apoptosis is considered to be a response to the $H$. pylori-induced mucosal damage.

Gastric epithelial cells proliferate in the lower part of the glandular neck, migrate up the crypt towards the surface, and then are shed into the lumen by apoptosis. In the present study, apoptosis was observed not only in 
the superficial epithelium but also in the proliferative zone of gastric mucosa with active inflammation, and the induction of apoptosis was markedly reduced by $H$. pylori eradication. The ammonia that accompanies $H$. pylori infection induces gastric epithelial cell apoptosis in vitro [15], and $C a g \mathrm{~A}$ and $V a c \mathrm{~A}$ proteins [16] are also considered to be influenced by epithelial cell apoptosis. Although it is still not known whether $H$. pylori affects apoptosis directly or indirectly, the epithelial cell apoptosis observed in $H$. pylori-infected mucosa is linked with cell proliferation. Our observations are compatible with those reported by Yabuki et al. [17].

Ki-67 immunohistochemistry and the TUNEL method have been employed as sensitive markers for evaluating the activation of proliferative cells and the acceleration of apoptosis in $\mathrm{H}$. pylori gastritis. The Ki67 labeling index has been reported to be generated by counting the number of positive cells per 300-3000 or more foveolar glandular cells in each biopsy sample [16-18]. However, one or two pathologists may select the fields, and count the Ki-67-positive cells by microscopy. Therefore, the data may not be objective and may be semi-quantitative at best. We used the image analysis processor to count the Ki-67-positive cells in this study. The IPAP provided a clear image for assessment. Thus, the IPAP may be a useful technology for evaluating the results of immunohistochemistry, and it could provide quantitative and reliable data for studying $H$. pylori gastritis.

Acknowledgments This work was supported in part by a grant from Otsuka Pharmaceutical Company (Tokyo, Japan) and by the Japanese Clinical Study Group of esophagocardiac lesion. We would like to thank Dr. Kaoru Hirabayashi for her helpful suggestion and Ms. Chiaki Sato-Matsuyama, Ms. Ayako Shimizu, and Ms. Takako Ohtsuki for their excellent technical assistance.

\section{References}

1. Correa P. Chronic gastritis: a clinicopathological classification. Am J Gastroenterol 1988;83:504-9.

2. Genta RM, Lew GM, Graham DY. Changes in the gastric mucosa following eradication of Helicobacter pylori. Mod Pathol 1993; 6:281-9.
3. Uemura N, Okamoto S, Yamamoto S, Matsumura N, Yamaguchi $\mathrm{S}$, Yamakido M, et al. Helicobacter pylori infection and the development of gastric cancer. N Engl J Med 2001;345:784-9.

4. Cahill RJ, Sant S, Beattie S, Hamilton H, O'Morain C. Helicobacter pylori and increased epithelial cell proliferation: a risk factor for cancer. Eur J Gastroenterol Hepatol 1994;6:11237.

5. Wotherspoon AC, Ortiz-Hidalgo C, Falzon MR, Isaacson PG. Helicobacter pylori-associated gastritis and primary B-cell gastric lymphoma. Lancet 1991;338:1175-6.

6. Fan XJ, Chua A, Shahi CN, McDevitt J, Keeling PWN, Kelleher D. Gastric T lymphocyte responses to Helicobacter pylori in patients with H. pylori colonization. Gut 1994;35:1379-84.

7. Fan XG, Kelleher D, Fan XJ, Xia HX, Keeling PWN. Helicobacter pylori increases proliferation of gastric epithelial cells. Gut 1996;38:19-22.

8. Karttunen R. Blood lymphocyte proliferation, cytokine secretion and appearance of $\mathrm{T}$ cells with activation surface markers in cultures with Helicobacter pylori. Comparison of the responses of subjects with and without antibodies to H. pylori. Clin Exp Immunol 1991;83:396-400.

9. Gerdes J, Lemke H, Baisch H, Wacker HH, Schwab U, Stein H. Cell cycle analysis of a cell proliferation-associated human nuclear antigen defined by the monoclonal antibody Ki-67. J Immunol 1984;133:1710-15.

10. Brenes F, Ruiz B, Correa P, Hunter F, Rhamakrishnan T, Fontham E, et al. Helicobacter pylori causes hyperproliferation of the gastric epithelium: pre- and post-eradication indices of proliferating cell nuclear antigen. Am J Gastroenterol 1993;88:18705 .

11. Watanabe T, Katsura Y, Yoshitake A, Masataki H, Mori T. IPAP: image processor for analytical pathology. J Toxicol Pathol 1994; 7:353-61.

12. Dixon MF, Genta RM, Yardley JH, Correa P. Classification and grading of gastritis - the updated Sydney system. Am J Surg Pathol 1996;20:1161-81.

13. Gavrieli Y, Sherman Y, Ben-Sasson SA. Identification of programmed cell death in situ via specific labeling of nuclear DNA fragmentation. J Cell Biol 1992;119:493-501.

14. Gerdes J. Ki-67 and other proliferation markers useful for immunohistological diagnostic and prognostic evaluations in human malignancies. Semin Cancer Biol 1990;1:199-206.

15. Tsuji S, Kawano S, Takei Y, Tsuji M, Kobayashi I, Nagano K, et al. Ammonia induces gastric cell apoptosis: possible implication to Helicobacter-related gastric mucosal atrophy (abstract). Gastroenterology 1995;108:244.

16. Moss SF, Calam J, Agarwal B, Wang S, Holt PR. Induction of gastric epithelial apoptosis by Helicobacter pylori. Gut 1996;38: 498-501.

17. Yabuki N, Sasano H, Tobita M, Imatani A, Hoshi T, Kato K, et al. Analysis of cell damage and proliferation in Helicobacter pyloriinfected human gastric mucosa from patients with gastric adenocarcinoma. Am J Pathol 1997;151:821-9.

18. Ikenaga M, Takano Y, Ohtani Y, Tsukamoto H, Hiki Y, Kakita A, et al. Low levels of apoptosis and proliferative activity in colorectal villous tumors: comparison with tubular tumors. Pathol Int 1998;48:453-9. 